\title{
Common hepatic artery arising from the aorta - demonstration with multidetector CT angiography and its clinical importance
}

\author{
Mustafa Koplay ${ }^{1}$, Mecit Kantarci
}

1Department of Radiology, Medical Faculty, Dumlupinar University, Kütahya, Turkey 2Department of Radiology, Medical Faculty, Atatürk University, Erzurum, Turkey

Submitted: 8 September 2009

Accepted: 9 October 2009

Arch Med Sci 2011; 7, 1: 176-177

DOI: 10.5114/aoms.2011.20628

Copyright (c) 2011 Termedia \& Banach

\author{
Corresponding author: \\ Mustafa Koplay MD \\ Department of Radiology \\ Medical Faculty \\ Dumlupinar University \\ The Central Campus \\ 43100, Kütahya, Turkey \\ E-mail: \\ koplaymustafa@hotmail.com
}

\begin{abstract}
We present a case of a rare variation of the hepatic artery discovered during the routine examination of abdomen by computed tomography. This variation was showed by multidetector-row computed tomography (MDCT) in a 35-yearold man. There have been shown the common hepatic artery arising from the anterior surface of abdominal aorta, $4 \mathrm{~mm}$ inferior to the celiac trunk. We discussed clinical significances of this variation during radiological procedures and surgical operations.
\end{abstract}

Key words: MDCT, hepatic artery, variation vascular, image.

We present a case of a rare variation of the hepatic artery (HA) discovered during routine examination of the abdomen by computed tomography (CT). This variation was identified in a 35 -year-old male patient suspected of renal artery pathology. The patient, who had a history of hypertension for three years, was examined with abdominal ultrasonography (US). Also colour Doppler US was performed in order to assess the renal arteries. US examination did not detect any pathology in our case. CT angiography was planned for a more detailed analysis of the renal arteries. Abdominal CT angiography was performed by a 16-detectorrow CT scanner (multidetector CT, Aquillon; Toshiba Medical Systems, Tokyo, Japan). Multidetector CT angiography revealed the common hepatic artery (CHA) arising from the anterior surface of the abdominal aorta, $4 \mathrm{~mm}$ inferior to the coeliac trunk (Figure 1). All the other vessels, organs, and systems of the patient were normal in every aspect, except this variation. There was no pathology of the renal arteries. Medical treatment was continued for hypertension.

Hepatic artery variation was first described by Michels et al. in the 1960s, and since then more and more variation types have been reported. Generally, the incidence of anatomical variations in the $\mathrm{HA}$ is $20-50 \%$, with diversity in different series [1]. Gruttadauria et al. [2] reported normal anatomy of the HA and variations of the $\mathrm{HA}$ in $57.8 \%$ and $42.2 \%$ of 701 cases, respectively. Some types of common variations were summarized, such as a replaced or accessory right $\mathrm{HA}$ arising from the superior mesenteric artery (SMA) (15\%), a replaced or accessory left HA originating 
from the left gastric artery (LGA) (11.6\%), and a replaced or accessory left HA stemming from the LGA and an accessory right HA originating from the SMA simultaneously (7.42\%). It was infrequent for a CHA to originate from the SMA $(0.86 \%)$ or be directed from the aorta $(0.43 \%)$. Being the rarest type of $\mathrm{HA}$ variations, a right $\mathrm{HA}$ might originate from the gastroduodenal artery or the aorta [1, 2]. According to the data in a study concerning 932 cases of liver transplantation, only $0.3 \%$ had the variation of the CHA arising from the aorta [3].

Hepatic artery variations can usually be explained by embryonic development. The liver is supplied during the fetal life by three arteries: the $\mathrm{CHA}$, the right HA from the SMA, and the left HA from the LGA. Anatomical variations correspond to the results of partial or complete persistence of the fetal pattern $[3,4]$.

The diagnostic methods for detecting hepatic arterial anatomy are catheter angiography, colour Doppler US, CT, and magnetic resonance (MR) angiography [5]. Catheter angiography is an invasive and expensive method which requires hospitalization of patients. Therefore, non-invasive techniques such as colour Doppler US, CT and MR angiography have become more widely used. Colour Doppler US may be difficult and time consuming when imaging in particular the distal part of the hepatic artery. In addition, it is an operatordependent examination with often limited value in obese patients. MR angiography is often of limited value when the patient is uncooperative, and it is not a preferred method due to its cost and prolonged scan time. In addition, because of movement artefacts, MR does not provide optimal imaging of the abdominal region. Smaller segmental vessels may not be consistently visualized with MR angiography [6]. With recent advances in CT technology, multidetector CT has replaced CA in the study of the hepatic arteries [5]. Multidetector $\mathrm{CT}$ is a reliable, easily applicable, and non-invasive tool for demonstration of abdominal organs and vascular structures.

The anatomy of the HA is of great importance in general surgery and hepatic surgery, especially in liver transplantation, as well as vascular radiology. Recently, the arterial vascular anatomy of the liver has played a significant clinical role, especially after the considerable development of hepato-pancreatobiliary surgery, the introduction of laparoscopic surgery, and the constant development of liver transplantation [2]. Knowledge of the potential HA anatomical variations is essential for a transplant surgeon in order to prevent vascular damage, and also in many radiological procedures such as transarterial chemo-embolization for hepatic tumours $[3,7]$. Multiplanar reconstruction and threedimensional (3D) volume rendering imaging fea-

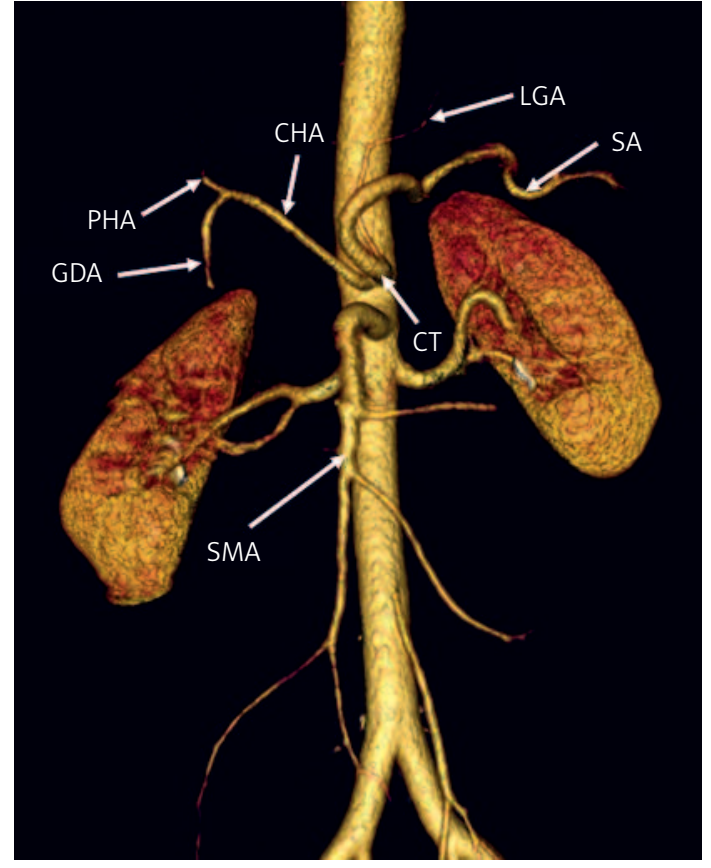

Figure 1. 3D volume rendering image shows the common hepatic artery arising from the anterior surface of the abdominal aorta

CT - coeliac trunk, CHA - common hepatic artery, PHA - proper hepatic artery, GDA - gastroduodenal artery, $L G A$ - left gastric artery, SA - splenic artery, SMA - superior mesenteric artery

tures of multidetector CT give important information about determination of these anatomical variations.

\section{References}

1. Xu X, Zheng SS. Variations and reconstruction of the hepatic artery in liver transplantation. Hepatobiliary Pancreat Dis Int 2006; 5: 170-2.

2. Gruttadauria S, Scotti Foglieni C, Doria C, Luca A, Lauro A, Marino IR. The hepatic artery in liver transplantation and surgery: vascular anomalies in 701 cases. Clin Transpl 2001; 15: 359-63.

3. Abdullah SS, Mabrut JY, Garbit V, et al. Anatomical variations of the hepatic artery: study of 932 cases in liver transplantation. Surg Radiol Anat 2006; 28: 468-73.

4. Osawa T, Feng XY, Sasaki N, et al. Rare case of the inferior mesenteric artery and the common hepatic artery arising from the superior mesenteric artery. Clin Anat 2004; 17: 518-21.

5. Saba L, Mallarini G. Multidetector row CT angiography in the evaluation of the hepatic artery and its anatomical variants. Clin Radiol 2008; 63: 312-21.

6. Sahani D, Mehta A, Blake M, Prasad S, Harris G, Saini S. Preoperative hepatic vascular evaluation with $C T$ and MR angiography: implications for surgery. Radiographics 2004; 24: 1367-80.

7. Uva P, Arvelakis A, Rodriguez-Laiz G, Lerner S, Emre S, Gondolesi G. Common hepatic artery arising from the left gastric artery: a rare anatomic variation identified on a cadaveric liver donor. Surg Radiol Anat 2007; 29: 93-5. 